\title{
SHP-1 sensitizes MCF-7 cells to trichostatin A-induced apoptosis by modulating PI3K-dependent events
}

\author{
Cell Death and Differentiation (2003) 10, 1213-1214. doi:10.1038/sj.cdd.4401292
}

\section{Dear Editor,}

Of the several signaling molecules important for cell survival, one that has garnered much attention is the serine/threonine kinase Akt. ${ }^{1}$ Downstream of phosphoinositide $3^{\prime}$-kinase (PI3K), Akt is activated by phosphorylation and regulates proapoptotic substrates, including Bad, caspase- 9 and the forkhead transcription factor, which are all inactivated by phosphorylation. $^{2-4}$ We have previously demonstrated that the p85 regulatory subunit of PI3K interacts with, and is negatively modulated by, the cytosolic protein tyrosine phosphatase SHP-1. ${ }^{5}$ SHP-1-mediated events are increasingly associated with genotoxic stress and, at least in our examination of somatostatin-induced apoptosis, appear to be dependent on caspase-8 recruitment, and increased p53 and Bax expression. ${ }^{6,7}$ Activation of caspases during apoptosis is a process tightly regulated by various $\mathrm{Bcl}-2$ family members.

The histone deacetylase (HDAC) inhibitors butyrate and trichostatin A (TSA) are gaining interest as potential anticancer drugs. Experimentally, TSA induces caspase activity and apoptosis in the MCF-7 breast cancer cell line via a cytochrome $c$-dependent pathway, ${ }^{8}$ thereby implicating caspase-9-dependent events. Interestingly, both butyrate and TSA can induce a shift in cellular tyrosine phosphorylation; one mechanism involves repression of mRNA and protein expression of the c-Src tyrosine kinase by inhibiting activity of each of its promoters, ${ }^{9}$ whereas the other mechanism involves induction of SHP-1 expression by increasing the activity of the tissue-specific $\mathrm{P} 1$ promoter in MCF-7 cells. ${ }^{10}$

We now demonstrate that the sensitivity of MCF-7 cells (HTB-22: ATCC) to TSA-induced apoptosis was heightened by stable overexpression of SHP-1, but not by overexpression of the catalytically inactive mutant of SHP-1, that is, SHP-1C455S. Expression of p85, the regulatory subunit of PI3K, in the MCF-7 parental cell line was transiently increased by TSA treatment indicating activation of cell survival mechanism(s). In contrast, this transient increase was absent in SHP-1-overexpressing cells and p85 immunodetection was, in fact, completely lost by TSA-treatment for $48 \mathrm{~h}$. Stable overexpression of SHP-1 during TSA treatment may induce an actual downregulation of total cellular p85 protein rather than a diminished phosphorylation of p85, which was proposed to account for the regulation of p85 activity by transient SHP-1 expression. ${ }^{5,11}$ The reduction in phosphorylation of Akt observed in TSA-treated SHP-1 transfectants was not observed in SHP-1-C455S transfectants, concurring with the observation that SHP-1, but not the catalytic inactive mutant, can negatively regulate p56 ${ }^{\text {Lck }}$ - induced phospho-PI3K activity and phosphorylation of Akt (Figure 1). ${ }^{11}$

Treatment with the tyrosine phosphatase inhibitor vanadate inhibits apoptosis by activation of PI3K/Akt, and phosphorylation of Bad. ${ }^{12,13}$ The present study revealed that basal phosphorylation of Bad-Ser136 was decreased by SHP-1 overexpression and further affected by TSA treatment. The basal and response levels of phospho-Akt were similarly affected in these same cells and, thus, support the notion that
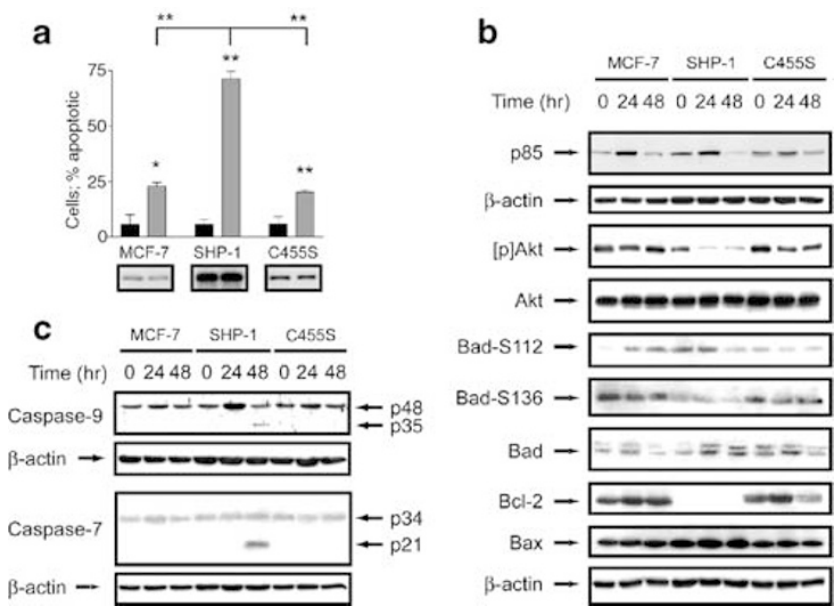

Figure 1 Effect of overexpressed SHP-1 on TSA-induced apoptosis in MCF-7 cell lines. (a) Neomycin-resistant pools of stable transfectants expressing wildtype SHP-1 [SHP-1] and the catalytic mutant SHP-1-C455S [C455S] were generated (the respective immunoblots are shown below the corresponding labels) and treated with TSA (300 $\mathrm{ng} / \mathrm{ml})$. Apoptotic events were assessed by fluorescence-activated cell sorting (using annexin-V-FLUOS; 10000 gated events/sample). MCF-7-SHP-1 transfectants were extremely sensitive to TSA treatment. The results (mean \pm S.D., $n \geq 3$ ) are presented as the percentage of apoptotic cells in control cultures (black bars) vs TSA-treated ( $48 \mathrm{~h}$ ) cultures (gray bars). *: $P<0.05$ and ${ }^{* *}: P<0.01$ (by ANOVA) vs the respective controls or between the indicated sampling groups. (b) TSA treatment induced a transient increase in expression of p85 in MCF-7 and a return to basal levels by $48 \mathrm{~h}$. Expression of p85 in similarly treated MCF-7-SHP-1 cells was almost completely abolished by $48 \mathrm{~h}$ of TSA treatment. Phosphorylation of Akt ([p]-Akt) was selectively decreased by TSA treatment in MCF-7-SHP-1 cells [SHP-1]. TSA also induced proapoptotic changes in the phosphorylation and/or expression of Bcl-2 family members, for example, Bad, Bcl-2 and Bax, almost exclusively in the MCF7 SHP-1 overexpressing cell line. (c) Cleavage of procaspase- 9 to the active p35 subunit was detected only in MCF-7-SHP-1 [SHP-1] cells at $48 \mathrm{~h}$ of TSA treatment. The MCF-7 cell line is caspase-3 null, but does express the executioner caspase-7, which, because of substrate recognition, is able to cleave the same substrates as caspase-3. A strong $21 \mathrm{kDa}$ band corresponding to activated caspase-7 cleaved from the $34 \mathrm{kDa}$ precursor was only detectable in MCF-7-SHP- 1 transfectants treated with TSA for $48 \mathrm{~h}$. Cleavage fragment sizes are indicated on the right. Representative blots probed for $\beta$-actin are included in (b) and (c) to confirm equivalent protein loading. 
phosphorylation of Bad-Ser136 is dependent on Akt kinase activation. $^{14}$ Basal phosphorylation of Bad-Ser112 was increased by SHP-1 overexpression, perhaps indicating a heightened cell survival response mechanism(s) to stress, and was diminished by TSA treatment of these cells. In contrast, increased phosphorylation of Bad-Ser112 was evident in TSA-treated MCF-7 parental cells, even though the phosphorylation of ERK $1 / 2$ in these same cells was greatly inhibited (data not shown). These combined data suggest either that phosphorylation of Bad-Ser112 is not as specific to ERK1/2 activation in MCF-7 cells as it has been demonstrated for other cell lines ${ }^{14}$ or simply that a redundancy in cell survival signaling pathways is revealed when ERK1/2 activation is compromised. What is germane to the present hypothesis is that SHP-1 overexpression leads to a loss of Bad phosphorylation (at Ser112 and Ser136) by $48 \mathrm{~h}$ of TSA treatment. It is well known that unphosphorylated Bad sequesters antiapoptotic molecules such as Bcl-2 away from the proapoptotic Bax and, once liberated, Bax translocates to the mitochondrial membrane where it modulates the formation of the permeability transition pore. This event allows intramembranal cytochrome $c$ to escape and to activate downstream caspases. The present observation of a complete ablation of $\mathrm{Bcl}-2$ protein by $\mathrm{SHP}-1$ overexpression concurrently with an increased expression of Bax protein would shift the cells' response from one promoting survival to one of a greater predisposition to an apoptotic outcome. This corroborates the angiotensin II-mediated (apoptotic) ERK inactivation, and the subsequent $\mathrm{Bcl}-2$ inactivation and $\mathrm{Bax}$ upregulation observed in PC12W and R3T3 cells. ${ }^{15}$ Our observation of proapoptotic changes in Bcl-2-related proteins coincides with increased sensitivity of MCF-7 cells to apoptogenic insult and the cleavage of caspase- 9 and of the executioner caspase-7. Processing of these caspases corresponded to a $170 \%$ increase in the activity of caspase-9 $\left[\mathrm{F}_{(3,15)}=3.775, P=0.0406\right]$ and a $160 \%$ increase in the activity of caspase-7 $\left[F_{(3,15)}=6.592, P=0.0070\right]$ (data not shown).

Perhaps what dictates sensitivity to apoptogenic stimuli is the level of expression of molecules such as Akt family members. It is known that AKT1/RAC $\alpha$ is overexpressed in less aggressive human breast cancer cell lines such as MCF$7,{ }^{16}$ whereas AKT3/RAC $\gamma$ is overexpressed in more aggressive forms of breast tumors. ${ }^{17}$ Our present data suggest that targeted manipulation of the Akt-dependent antiapoptotic signaling pathway in combination with a relative shift in cellular protein tyrosine phosphatase activity may provide a novel approach to enhancing the efficacy of adjuvant therapies in the clinic.

\section{$Y X u^{1,2}$, DD Mousseau ${ }^{*}{ }^{, 1,4}$, D Banville ${ }^{1}, X$ Zhao $^{2}$ and S-H} Shen $^{*}, 1,3$

${ }^{1}$ Mammalian Cell Genetics, Health Sector, Biotechnology Research Institute, 6100 Royalmount Ave., Montreal, Quebec, Canada H4P 2R2

2 Department of Animal Science, Macdonald Campus, McGill University, Ste. Anne de Bellevue, Quebec, Canada H9X 3V9

${ }^{3}$ Department of Medicine, McGill University, Montreal, Quebec, Canada H3G $1 \mathrm{~A} 4$

${ }^{4}$ Current address: Neuropsychiatry Research Unit, Department of Psychiatry, University of Saskatchewan, Saskatoon, Saskatchewan, Canada, S7N 5E4.

* Corresponding authors: DD Mousseau, E-mail: darrell.mousseau@usask.ca and SH Shen: E-mail: Shi.Shen@cnrc-nrc.ge.ca

1. Datta SR et al. (1999) Genes Dev 13: 2905-2927

2. Datta SR et al. (1997) Cell 91: 231-241

3. Cardone MH et al. (1998) Science 282: 1318-1321

4. Brunet A et al. (1999) Cell 96: 857-868

5. Yu Z et al. (1998) J. Biol. Chem. 273: 3687-3694

6. Thangaraju M et al. (1999) Cancer Res. 59: 1649-1654

7. Liu D et al. (2000) J. Biol. Chem. 275: 9244-9250

8. Medina V et al. (1997) Cancer Res. 57: 3697-3707

9. Kostyniuk CL et al. (2002) Oncogene 21: 6340-6347

10. $\mathrm{Xu} Y$ et al. (2001) Gene 269: 141-153

11. Cuevas B et al. (1999) J. Biol. Chem. 274: 27583-27589

12. Chin LS et al. (1999) J. Biomed. Sci. 6: 213-218

13. Lawson AE et al. (2000) Blood 96: 2084-2092

14. Hayakawa J et al. (2000) Cancer Res. 60: 5988-5994

15. Horiuchi $\mathrm{M}$ et al. (1998) Endocr. Res. 24: 307-314

16. Jones PF et al. (1991) Cell Regul. 2: 1001-1009

17. Nakatani K et al. (1999) Biochem. Biophys. Res. Commun. 257: 906-910 